\title{
Managing Decisions on Changes in the Virtual Enterprise Evolution
}

\author{
Marcus Vinicius Drissen-Silva ${ }^{1}$ and Ricardo José Rabelo ${ }^{2}$ \\ ${ }^{1}$ Postgrad. Program of Electrical Engineering, Federal University of Santa Catarina, Brazil \\ ${ }^{2}$ Department of Automation and Systems, Federal University of Santa Catarina, Brazil \\ GSIGMA - Intelligent Manufacturing Systems Group \\ drissen@das.ufsc.br, rabelo@das.ufsc.br
}

\begin{abstract}
VE evolution deals with problems that happen during the VE operation and that put on risk planned results. This requires the application of problem-solving mechanisms to guarantee the construction of a new but feasible VE plan. Grounded on Project Management and Decision Support Systems foundations, this paper proposes a distributed collaborative decision support system to manage the VE evolution. Its main rationale is that VE's members are autonomous and hence that all the affected partners should discuss about the necessary changes on the current VE's plan. In the proposed approach, this discussion is guided by a decision protocol, and the impact of decisions can be evaluated. Results of a first prototype implementation are presented and discussed, with a special focus on the part which regulates the argumentation, voting and comparison of possible solutions.
\end{abstract}

Keywords: Collaborative discussion, Decentralized Decision-making, Project Management, Management of Changes, Virtual Enterprises.

\section{Introduction}

Collaboration between companies is an increasing business strategy to face the global competition. Collaborative Networks (CN) offer conditions to companies to reduce expenses, increase capacity, broaden markets and improve themselves with knowledge acquired in business [1]. There are several manifestations of CN. This paper focuses on CNs of type Virtual Enterprises (VE).

A VE is seen as a dynamic, temporary cluster of autonomous enterprises that collaborate with each other to attend a given business opportunity or to cope with a specific need, where partners share risks, costs and benefits, and whose operation is achieved by a coordinated sharing of skills, resources, information and knowledge, mostly supported by computer networks [2], offering services abroad as if it were a single organization [1].

A VE is typically organized in a number of phases known as VE lifecycle: creation, operation, evolution and dissolution phases [3]. In very general terms, the creation phase comprises VE planning and partner's selection; the operation phase comprises the execution and monitoring of the planned activities; the evolution phase comprises the handling of problems detected in the operation phase; and the dissolution phase comprises all issues associated to the VE ending. This paper focuses on the 
VE evolution phase. It also assumed that companies belong to a VBE (Virtual Organization Breeding Environment) [4], so having common operating principles, rules and the required basis of trust among them.

In the evolution phase, managers have to deal with the need of changes in the current VE's plan, considering the different type of problems that may happen along its execution. Examples of problems include: anticipation or delay in item's delivery; low partner's performance; tasks not properly accomplished; changes in the product's specification; and logistics problems.

Unlikely other types of CNs, like supply chains or extended enterprises, the managing of the VE evolution implies the consideration of relevant additional aspects [5]: partners are totally autonomous so decisions cannot be imposed by a single company; they have different cultures and use different organizational practices; the decision process should be transparent regarding governance aspects; the information necessary to help solving the problem should be disclosed and shared taking into account temporary and current access rights; enterprises are often involved in several VEs simultaneously, where some of them are inter-related. Besides that, a VE is something very much unique, which means that the way a problem was solved in a given previous VE is not necessarily valid for another VE.

Therefore, managing the evolution of a VE requires an ample spectrum of issues that make decision-making extremely complex. As most of VE members are composed of SMEs, whose limitations are very known, this difficulty is even harder.

In this sense, this paper presents some results of an ongoing work which aims at developing a collaborative, flexible and human-centered decision support framework to help VE members in the management of problems that cause changes in the VE execution, considering those mentioned requirements.

This paper is organized as follows: Section 1 presented a general analysis of the requirements for VE management and the evolution phase. Section 2 discusses the distributed approach for management the VE evolution as well as points out some of the involved requirements. Section 3 introduces the proposed framework. Section 4 presents some current results of the framework. Section 5 provides a preliminary assessment and the next short-term steps.

\section{Managing the VE Evolution}

VE management "designates arrangement, allocation and coordination of the resources and their tasks, as well as their inter-organizational premises, to the VE goals, respecting time, cost and quality" [6]. This general definition can embrace diverse perspectives of analysis on how such management can be concretely done.

One can note that developing a comprehensive and flexible environment that can cope with all the mentioned requirements for the management of the VE evolution phase is indeed challenging, both in terms of managerial methods and models, and from the IT point of view. Some authors have approached this problem (including the operation phase) in different ways. For example, Rabelo and Pereira-Klen [7] have introduced a fixed decision protocol to deal with changes in the VE. Hodík and Stach [8] have developed a multi-agent-based decision support system to simulate the impact of decisions. Negretto et al. [9] have created a distributed supervision system to monitor the VE plan. Noran [10] has developed a decision support framework to help managers in the partners' selection in the VO creation. 
Besides leaving only to the VE coordinator all the rights to access information and to take decisions, these works seem to be not so suitable to cope with two key requirements of $\mathrm{VE}$ evolution: the decentralization of decision-making and partners' autonomy. Considering the new requirements arisen with the VE concept, it seems unrealistic to apply a centralized approach where only one company can decide and impose to the others a solution for a problem. Finding a solution for a problem in this scenario is very complex as this involve many particular details and require several constraints analysis and relaxations. Thus, it is even dangerous to leave the decision only to the VE coordinator as the ultimate goal comprehends reaching a feasible and realistic solution, and not just another theoretical VE plan. Instead, partners should discuss about the problem, and the solution should emerge from this respecting their autonomies, leading to a decentralized and distributed scenario for decision-making.

Distributed decision-making is not a new research topic, and many works have been developed along the last decade concerning this matter, especially in the form of distributed decision support systems [11]. Despite its theoretical adequacy, one of the critical and practical problems of this approach is that it intrinsically imposes the need for having several discussions on the network, which use to be not so efficient, it is easy to lose the focus, and it depends too much of the quality of the communication and security infrastructures. On the other hand, this seems to be an intrinsic price to pay.

This paper presents a distributed approach to tackle the VE evolution requirements but where those problems are mitigated. In order to better understand the approach's rationale, it tries to implement the following vision:

"Partners, although being distributed and autonomous, belong to a VBE so they share common operating principles. One of them is that they trust on each other and they should collaborate towards reaching a globally feasible solution for a problem in the VEs they are involved in. They should discuss about it through the Internet, making use of an open distributed decision-making environment. The discussions should be structured and guided in order to keep the focus on the problem and to have a potentially better decision. This structure should be connected to the affected business processes (context), and not treated as an isolated event. It should be also flexible and adaptive regarding the usual uniqueness of the problem and of the VE so that partners can have some freedom to exchange ideas while they evaluate possibilities against their availabilities. This evaluation should be made via an easy access to common managerial software tools in order to facilitate SME managers' actions. After this, they should have means to evaluate the impact of their decisions before acting. All this should be supported by adequate ICT infrastructures, which can also provide the necessary security in the communications and access to information."

This vision imposes, however, a new set of (functional) requirements:

- Partners' discussion: this is related to endowing partners with a collaborative environment where they can exchange information towards the problem resolution. Groupware tools [12] have been largely used nowadays to support multiple users working on related tasks in local and remote networks. This can also involve supporting tools for argumentation, like HERMES [13], methods to help finding consensus on topics of discussion, like DELPHI [14], and integrated and secure web-based groupware services devoted to CNs, like the one developed by Woelfel et al. (described by Rabelo et al. [15]). 
- Methodological guidance: the use of a methodology has the aim of preventing partners from dealing with the problem without any guidance, making them losing time and resources, which in turn can hazard the VE's business. An approach for that is to see a VE as a project, making use of project management reference models, like PMBOK [16] or ECM Engineering Change Management [17].

- Modular and flexible execution of decision protocols: decision protocols can be seen as an instrument to: i) systemize a set of actions where there is a strong human intervention, ii) standardize and iii) enhance their execution efficiency [7]. The idea is to provide a base protocol where flexible configurations can be made considering the particularities of each VE.

- Performance measurement and monitoring: This issue looks to the current situation of the production system to further offer conditions for VE partners measuring their own performance as well as checking their capacity to get more confidence when deciding. This can involve traditional models/techniques, as BSC Balanced Scorecard [18], SCOR - Supply Chain Operation Reference [19], and OLAP - On-line Analytical Processing [20], as well as the definition of the most adequate performance indicators that should be applied to the given case and members, as proposed in Baldo et al. [21].

- Performance evaluation: the goal of this aspect in the context of VE evolution management is to provide partners with techniques that help them to evaluate the impact of their decisions at their companies along the discussion process. At the same time, it allows the VE coordinator to evaluate the global solution before validating the final decision. These techniques can be based on analytical modeling, simulation, or direct measurement [22].

- Knowledge Inheritance Mechanisms: VE inheritance is considered as a process of transmitting the information and the knowledge acquired in the past experiences for future collaborative organizations [23]. The role of inheritance mechanisms is to help managers to improve their decisions when considering the lessons learned in the past.

- Business Auditing: auditing is crucial to keep the trust in the whole decision environment alive. When managers get together to discuss about a given problem, they exchange opinions, share data and take decisions. In this way, it is necessary to integrate information for further auditing (especially considering contractual non-conformity), giving the necessary transparency to the process [24].

- ICT Infrastructure: Information and Communication Technology (ICT) Infrastructures are a mean to support the main transactions among partners in a $\mathrm{CN}$. Actually, this is one of the conditions to work as such [15]. In the context of VE evolution and decision-making, a secure ICT infrastructure is responsible for supporting all the mentioned functionalities.

In way to instantiate the presented vision and transforming these new requirements into more concrete artifacts and integrated framework, the purpose of this work is to provide a supporting framework and methodology that can systematize, guide and 
assist VE managers in the discussions about a specific problem towards its resolution, within the VE evolution phase. This methodology is represented by a decision protocol. It corresponds to decision blocks, grouped in a macro sequence of actions, which are instantiated according to the various specificities of each VBE, VE and types of problems, considering the set of requirements previously mentioned. Next section depicts this proposal.

\section{Decision Support Framework}

Previous section has presented the main aspects needed to support decision on changes in the VE evolution according to the authors' vision. In order to cope with this, a framework has been conceived. This framework gathers such aspects and groups them into four pillars: Human, Organizational, Knowledge and Technological. The essential rationale of these four pillars is to enable (empowered) humans to discuss and to decide about a problem related to a given organizational process, applying a set of organizational procedures and methods, using information and knowledge available in the VBE's data repositories, all this supported by a sort of ICT (technological) tools and infrastructures [5]. The discussion is framed by a decision protocol and it is carried out within a distributed and collaborative decision support environment. The decision protocol is the mechanism which links the four pillars according to each problem in the VE evolution. Figure 1 shows the framework.

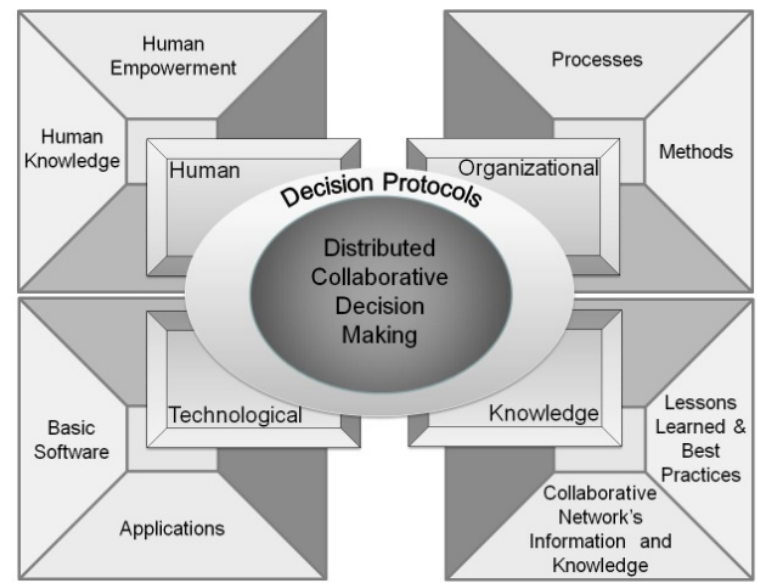

Fig. 1. Framework for VE Evolution Management

The Human pillar represents people, i.e. the VE companies' managers who use their tacit knowledge and collaborative attitude to help solving the problem come from the VE operation. The Organizational pillar comprises intra and inter-enterprises processes, ontologies, working methods, techniques and procedures that should be involved in the envisaged distributed and collaborative decision-making process. The Knowledge pillar comprises explicit information and knowledge available in the VBE's data repositories. The Technological 
pillar refers to all kind of ICT tools, platforms and security artifacts available to help managers in the access to the organizational working methods.

\subsection{Framework Architecture}

The four framework's pillars are operated through three concrete elements: the decision protocol, the distributed and collaborative decision support computing environment, and the ICT Toolbox. They all form the Distributed Collaborative Decision Support System for the Management of VE Evolution (DDSS-VE). Based on the classification proposed by Turban and Aronson [26], DDSS-VE can be classified as a negotiation-based, decentralized, partially hierarchical, semi-structured, multiparticipant and team-based system.

Figure 2 presents the framework's architecture, also illustrating the relation of the elements with the pillars.

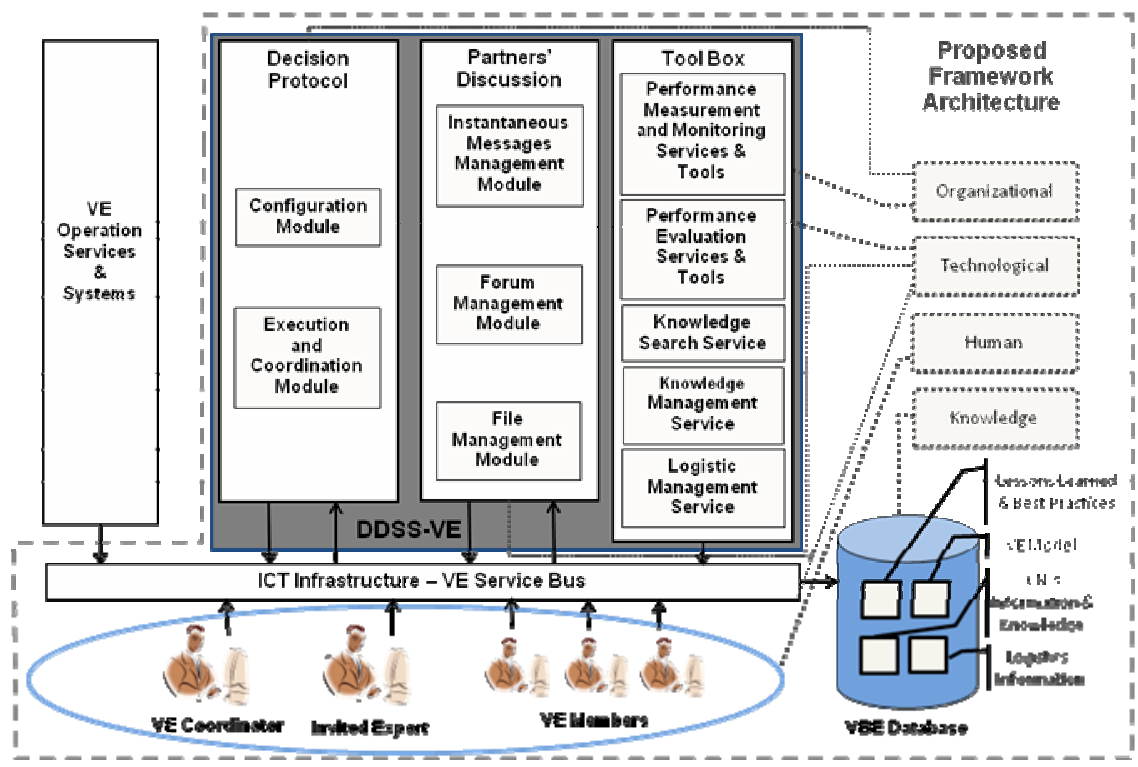

Fig. 2. Framework Architecture

VE operation services \& systems represent the activities responsible for monitoring and detecting problems in the current VE's plan. Once a problem is detected, the control flow is then passed to the DDSS-VE in order to manage the problem resolution. There are three main modules involved in the DDSS-VE architecture. The Decision Protocol is responsible for guiding and coordinating the discussions among partners, also considering the set of (configured) particularities of the VE. The Discussion Environment is responsible for supporting the discussion itself among the VE partners (VE Coordinator, the VE members and, optionally, helped by an invited expert). It is composed of an instant message module (a Chat), a forum module and a file exchange module, where partners can discuss, argument and exchange information during the 
problem resolution. The Tool Box contains a set of tools and software services to help partners in the discussions and evaluations. It is composed of performance monitoring and evaluation tools and other supporting services. ICT infrastructure acts as the bus that integrates all these modules, tools and services as well as that grants access to the VBE database. These three modules are more detailed in the next sections.

\section{Decision Protocol}

The decision protocol is a sequence of steps which defines the activities that have to be executed in given situations within a given context to solve a problem. Its rationale was mentioned in section 2, and it is detailed explained in Drissen-Silva and Rabelo [5]. Conceptually, it should indicate what has to be done, why, by whom, where, when, how, and with which resources. In this work, it is the most important element as it governs the whole process.

This protocol intends to help managers in the decision-making process, showing them what have to be done in a proper moment. Figure 3 shows the proposed decision protocol. It is strongly based on the ECM reference model's phases [17] and O'Neill's model [25], but adapted to the VE evolution context. All steps are modeled as business processes using BPMN (Business Process Modeling Notation), which offers the required protocol's flexibility for being adapted to each different scenario and VE topology or objective. By means of a Business Process Management (BPM) tool, process execution (i.e. the protocol steps) can be monitored.

A problem is not solved at once. It requires the resolution of many sub-problems, which in turn can demand diverse rounds of exchange of information, computer-aided analysis and managers' opinions. The process ends when a considered good solution is achieved. After this, the control flow goes back to the VE Operation. The discussions and decisions are stored for future evaluation and eventual auditing. In the case no solutions are found out, the situation is sent to the tactic and strategic levels (which are out of scope of the proposed model) in order to, for example, renegotiate the delivery date with the customer.

\section{Partner's Discussion Environment}

After the problem has been detected, DDSS-VE starts the protocol steps (Figure 3), within the Need of Change Identification phase. The affected partners are identified and can be invited to participate in the collaborative discussion and decision-making. In the Change Proposal phase, the discussion is supported by services that combine the HERMES system's approach with Delphi method (see section 2). The part inspired in HERMES aims to organize partners' arguments in a concise structure, using an appropriate semantic, communicating their suggestions but in a organized way, including an association of weights to the most important arguments. This aims at finding a consensus about the problem resolution. The part inspired in Delphi aims at avoiding direct confrontations among participants, which could generate counterproductive discussions. All the arguments are gathered by the VE Coordinator who, at the first moment, acts as the moderator, selecting, deleting, changing or suggesting changes in the received arguments before they can become available to the others.

The Toolbox. Traditionally, SMEs have many difficulties to access, use and maintain software, mainly due to its costs and to the required staff to maintain it. The proposed architecture has been conceived to work under the on-demand approach, where 


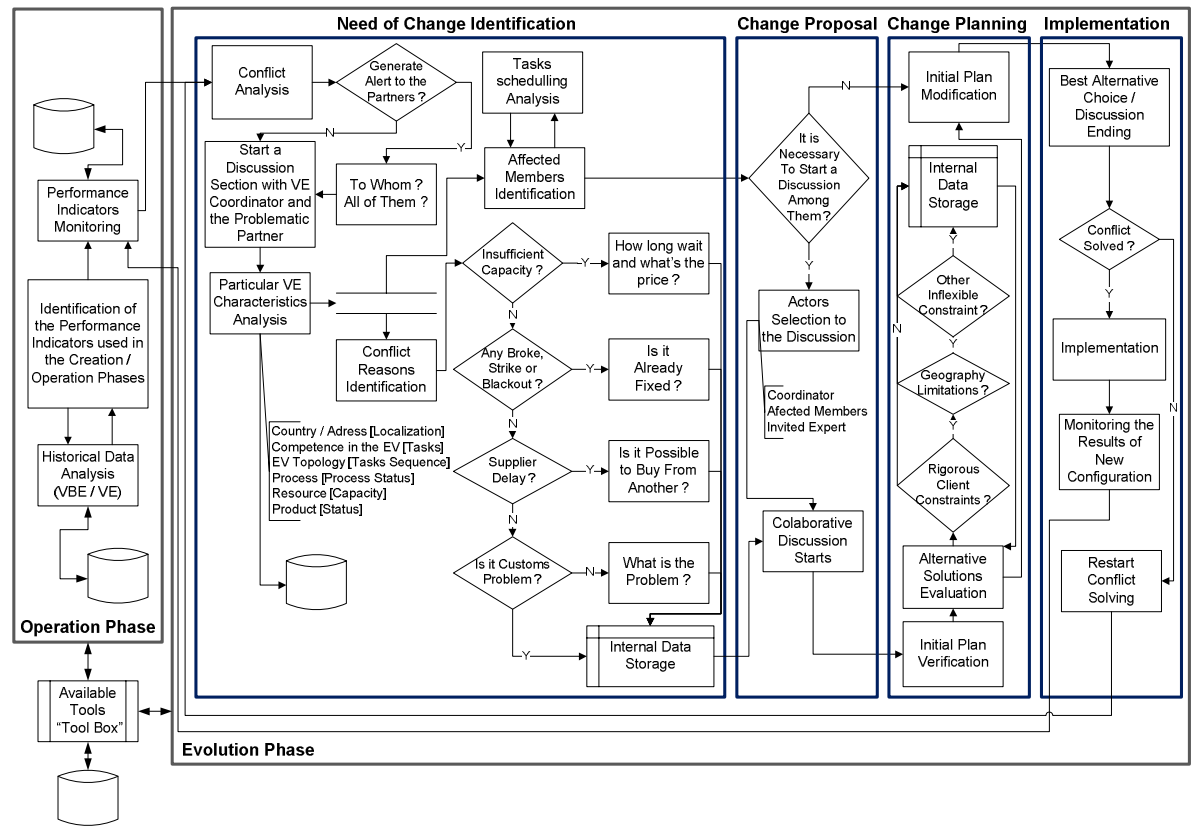

Fig. 3. Basis Protocol for the VE Evolution Management

software services and tools are accessed remotely and only when necessary. Rabelo et al. [15] have developed a secure ICT infrastructure devoted to CNs where the access to it is totally made in the form of services, following the SOA (Service Oriented Architecture) paradigm. The current version of this infrastructure only covers the VE creation [27] and operation phases [9]. The DDSS-VE intends to act as a service for the VE evolution within a wider and comprehensive framework for VE management. Other supporting software, like simulators and spread sheets, are to be accessed under the ASP (Application Service Provider) model. ICT Toolbox is therefore a logical repository of common tools that are accessed via the ICT infrastructure / Internet, facilitating members' acceptance and use of management methods. This however does not cover the existing local tools used by each member at their companies.

\section{Prototype Implementation}

This section presents current results of the implementation of the DDSS-VE framework, which is now concentrated on the Partners' Discussion Environment part. So far it assumes the existence of some of the architecture's elements, namely the VBE database, the ICT infrastructure, and some basic Toolbox's services. Within a controlled testing environment, the problems detected in the VE operation phase are manually introduced and the discussions are simulated in a distributed scenario using a number of PCs. The implementation of supporting simulation tools is not yet done, with the prototype currently assuming that members have this support. 
In this first implementation phase, the goal has been to combine HERMES system and Delphi method, and to adapt them to the desired decision philosophy. In other words, it aimed at facing the partners' autonomy and transparency requirements as well as the need for a more structured way of deciding. The main adaptations include:

- The creation of a moderator (role), who is responsible to evaluate and to make available the arguments sent by members. Depending on the case, the moderator can be the own VE coordinator.

- The comparison of two different arguments using different connectors (better than; worse than; equal to; as bad as; as good as). Each comparison assigns negative and/or positive points to each argument, depending on the connector.

- Voting. Partners can vote pro or against to each argument.

\subsection{Usage Scenario}

In order to evaluate the current implementation stage of the DDSS-VE, a VE scenario has been created. This hypothetic VE would be responsible to develop a new helmet style for racing, involving four partners from different countries.

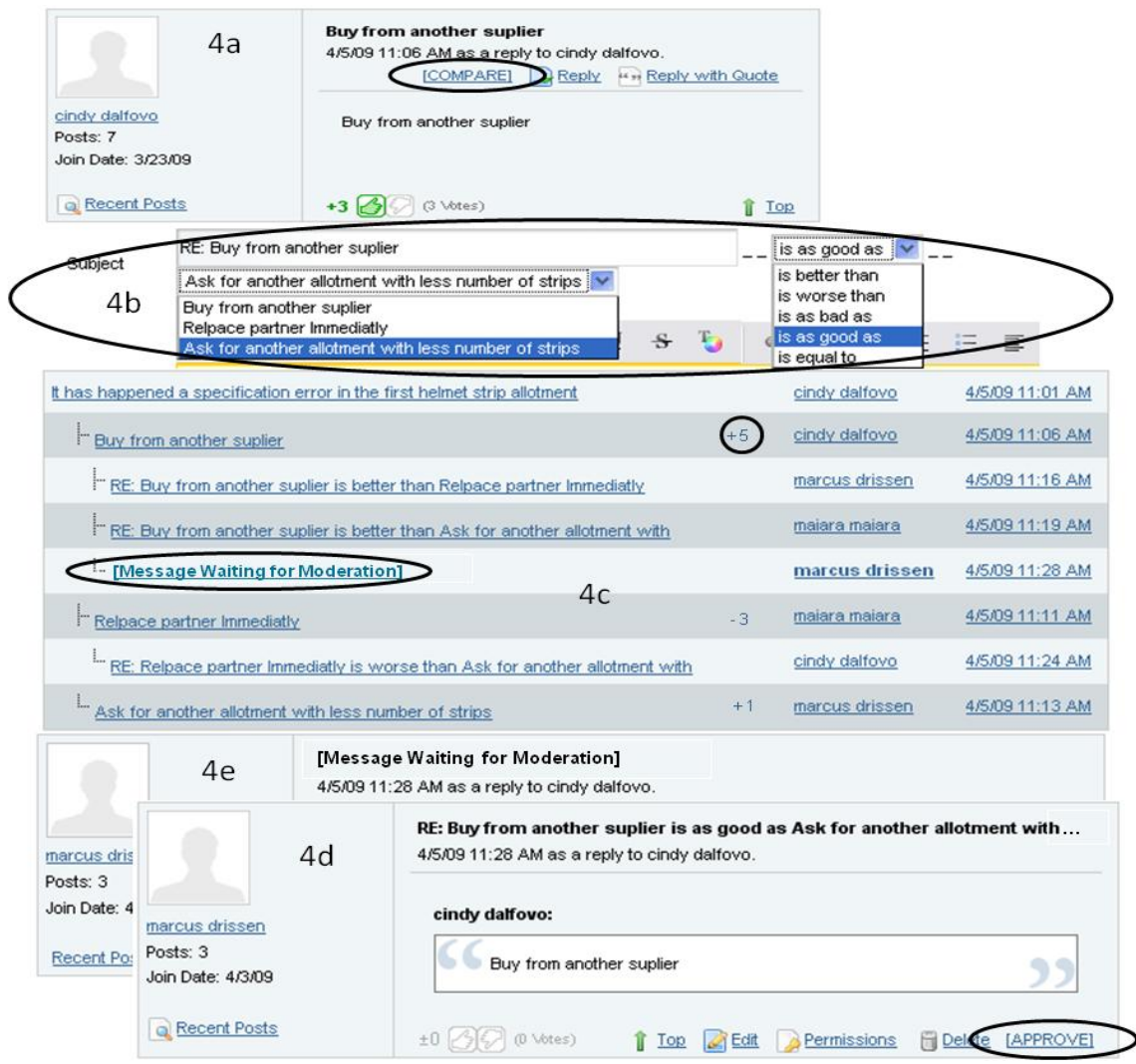

Fig. 4. Some snapshots of the Partner's Discussion Environment 
Considering the decision protocol showed in figure 3, it is assumed that the phase "Need of Change Identification" has been passed (as the current implementation does not focus on it). The steps below and figure 4 illustrate in a general way (due to size restrictions) how the protocol would conduct the conflict resolution from the phase "Change Proposal" on. All this have been implemented in a web portal, on top of Liferay web application server (www.liferay.com). In this example, the VE Coordinator (Ms. Cindy) has concluded that it is necessary to start a discussion with two members (Mr. Marcus and Ms. Maiara) due to a problem detected in the specification of the first lot. After starting the collaborative discussion, the protocol gets in the "Changing Planning" phase and ends (in terms of current implementation stage) when the best alternative has been chosen, in the "Implementation" phase:

\section{Starting the discussion (to be conducted via the DDSS-VE):}

- The protocol ask some questions to delineate the better attitude for each case (e. g. if it is a rigorous client constraint that avoids from choosing another supplier);

- $\quad$ Each participant will use some tools to preview which different scenarios could be acceptable to reschedule the activities that have to be done, choosing the best one, and publishing it as a suggestion for the problem resolution:

a. Ms. Cindy posts the first suggestion: 'Buy from another supplier' (Figure 4a);

b. Each partner can vote pro or against it (bottom Figure 4a);

c. Each suggestion can be compared with other suggestions using 'COMPARE' button (Figure $4 a$ ). Figure $4 b$ presents the list of suggestions and the possible logical connectors. For example, a comparison using 'is better than' as the connector assigns +1 point to the best suggestion and -1 to the worst;

d. Figure $4 c$ shows a tree (associated to the detected problem: helmet strip allotment) with the three posted suggestions (plus authors) and four comparisons among them. One of them is not yet evaluated as it is 'waiting for moderation';

- The moderator (Ms. Cindy) evaluates the different suggestions and the comparisons, mainly to see if there is some confrontation among the participants. In this case, there is no confrontation as there is not any additional text below the text between quotes in the box, which is the initial moderator's suggestion to solve the problem:

a. Figure $4 d$ shows the Moderator's view. She can modify and/or simply approve Mr. Marcus' opinion ("RE: buy from another supplier is as good as ...") about her initial proposal (expressed between quotes: 'buy from another supplier') and send them to the group;

b. Figure $4 e$ represents the vision seen by the other two members before Mr. Marcus' opinion approval. Thus, they only see 'message waiting for moderation';

- In what the final voting result is concerned:

a. It is possible to see the number of votes of each suggestion, which is +3 in relation to the Ms. Cindy's one (Figure 4a), also meaning that the three consulted members (including the VE coordinator) have agreed on it.

b. Figure $4 c$ shows a signaled number beside each suggestion expressing the final sum of voting with the weights of comparisons. In this case, 'Buy from another supplier' has more positions in favor (+5): 3 from direct voting and 2 from two comparisons. 
2. Once agreed, the most suitable solution is settled on the VE plan and partners (re)start to work based on it. This means that VE evolution is ended and the VE management goes back to the operation phase.

\section{Conclusions}

This paper has presented a framework to support a collaborative discussion among VE members for solving problems during the VE evolution phase. It is essentially composed of a decision protocol, a distributed and collaborative decision support system, and of ICT supporting tools and communication infrastructure. It has been designed to cope with the VE requirements, mainly in what members' autonomy and decision transparency is concerned.

Developed based on project management methodologies, discussions are guided and assisted by the system but preserving and counting on the members' experience and knowledge in order to reach a suitable/feasible solution for the given problem.

Current implementation results have preliminary showed that the proposed mechanisms for supporting partners' autonomy, Internet-based decentralized decision-making, voting and transparency have worked out in a controlled environment. During the discussions, selected partners can have access to the problem, can freely exchange opinions about how to solve it, and can express their preferences via voting. This guarantees that the solution emerges from the collaboration and trust among partners.

This is an ongoing work. This paper has depicted the framework and the implementation of one of its parts, which is the discussion environment. Next short-term steps refer to: i) the implementation of the decision protocol part and; ii) its integration with the available toolbox's services (mainly the ones that will help in the evaluation of the changes impact on each local member).

Acknowledgments. This work has been supported by CNPq - The Brazilian Council for Research and Scientific Development (www.cnpq.br). The authors would like to thanks Ms. Cindy Dalfovo for the software implementation activities of this work.

\section{References}

1. Camarinha-Matos, L.M., Afsarmanesh, H., Ollus, M.: ECOLEAD: A Holistic Approach to Creation and Management of Dynamic Virtual Organizations. In: Camarinha-Matos, L.M., Afsarmanesh, H., Ortiz, A. (eds.) Collab. Networks and Their Breeding Environments, pp. 3-16. Springer, Heidelberg (2005)

2. Rabelo, R.J., Pereira-Klen, A.A., Klen, E.R.: Effective management of dynamic supply chains. Int. J. Networking and Virtual Organisations 2(3), 193-208 (2004)

3. Camarinha-Matos, L.M., Afsarmanesh, H.: The Virtual Enterprises Concept. In: Camarinha-Matos, L.M., Afsarmanesh, H. (eds.) Infrastructures for Virtual Enterprises: networking industrial enterprises, pp. 3-14. Kluwer Academic Publishers, US (1999)

4. Afsarmanesh, H., Camarinha-Matos, L.M.: A Framework for Management of Virtual Organization Breeding Environments. In: Proceedings 6th IFIP Working Conf. on Virtual Enterprises, pp. 35-48. Kluwer Acad. Publishers, Dordrecht (2005) 
5. Drissen-Silva, M.V., Rabelo, R.J.: A Collaborative Decision Support Framework for Managing the Evolution of Virtual Enterprises. International Journal of Production Research 47(17), 4833-4854 (2009)

6. Jansson, K., Eschenbaecher, J.: Challenges in Virtual Organisations Management - Report on methods for distributed business process management. Tech. Report D32.1. European Collaborative networked Organizations LEADership initiative. FP6 IP 506958 (2005)

7. Rabelo, R.J., Pereira-Klen, A.A.: A Multi-agent System for Smart Co-ordination of Dynamic Supply Chains. In: Proceedings PRO-VE 2002, pp. 312-319 (2002)

8. Hodík, J., Stach, J.: Virtual Organization Simulation for Operational Management. In: 2008 IEEE CSM Int. Conf. on Distributed Human-Machine Systems, Czech Technical University in Prague (2008) ISBN 978-80-01-04027

9. Negretto, H., Hodik, J., Mulder, W., Ollus, M., Pondrelli, P., Westphal, I.: VO Management Solutions: VO management e-services. In: Camarinha-Matos, L.M., Afsarmanesh, H., Ollus, M. (eds.) Methods and Tools for Collab. Netwoked Org., pp. 257-274. Springer, Heidelberg (2008)

10. Noran, O.: A Decision Support Framework for Collaborative Networks. In: CamarinhaMatos, L.M., Afsarmanesh, H., Novaes, P. (eds.) Establishing the Foundation of Collaborative Networks, pp. 83-90. Springer, Heidelberg (2007)

11. Bostrom, R., Anson, R., Clawson, V.: Group facilitation and group support systems. Group Support Systems: New Perspectives. Macmillan, Basingstoke (2003)

12. Wulf, V., Pipek, V., Won, M.: Component-based tailorability: Enabling highly flexible software applications. Int. Journal Human-Computer Studies 66(1), 1-22 (2008)

13. Karacapilidis, N., Papadias, D.: Computer supported argumentation and collaborative decision making: the HERMES system. Information Systems 26(4), 259-277 (2001)

14. Dalkey, N.C., Helmer, O.: An experimental application of the Delphi method to the case of experts. Management Science 9, 458-467 (1963)

15. Rabelo, R.J., Castro, M.R., Conconi, A., Sesana, M.: The ECOLEAD Plug \& Play Collaborative Business Infrastructure, in Methods and Tools for Collab. In: Camarinha-Matos, L.M., Afsarmanesh, H., Ollus, M. (eds.) Networked Org., pp. 371-394. Springer, Heidelberg (2008)

16. PMBOK.: A Guide to the Project Management Body of Knowledge. PMI Standards Committee (2004)

17. Tavčar, J., Duhovnik, J.: Engineering change management in individual and mass production. Robotics and Computer-Integrated Manufacturing 21(3), 205-215 (2005)

18. Kaplan, R.S., Norton, D.P.: The Strategy in Action, Rio de Janeiro: Campus (1997)

19. SUPPLY_CHAIN_COUNCIL.: Supply Chain Operations Reference Model - SCOR Version 7.0 Overview (2005)

20. Moon, S.W., Kim, J.S., Kwon, K.N.: Effectiveness of OLAP-based cost data management in construction cost estimate. Automation in Construction 16(3), 336-344 (2007)

21. Baldo, F., Rabelo, R.J., Vallejos, R.V.: Modeling Performance Indicators' Selection Process for VO Partners' Suggestions. In: Proceedings BASYS 2008 - 8th IFIP Int. Conf. on Information Technology for Balance Automation Systems, pp. 67-76. Springer, Heidelberg (2008)

22. Jain, R.: The Art of Computer Systems Performance Analysis: Techniques for Experimental Design, Measurement, Simulation and Modeling. J. Wiley \& Sons, Inc., Chichester (1991)

23. Loss, L., Pereira-Klen, A.A., Rabelo, R.J.: Virtual Organization Management: An Approach Based on Inheritance Information. In: Global Conference on Sustainable Product Development and Life Cycle Engineering, São Carlos, SP, Brazil, October 3-6 (2006) 
24. Gil, A.L.: Business Auditing. Governmental Auditing - Contingences versus Quality, 2nd edn. São Paulo, Atlas (2002) (in Portuguese)

25. O'Neill, H.: Decision Support in the Extended Enterprise, Ph.D. Thesis, Cranfield University, The CIM Institute (1995)

26. Turban, E., Aronson, J.: Decision support systems and intelligent systems. A Simon and Schuster Company, Upper Saddle River (1998)

27. Afsarmanesh, H., Msanjila, S., Erminova, E., Wiesner, S., Woelfel, W., Seifert, M.: VBE Management System. In: Camarinha-Matos, L.M., Afsarmanesh, H., Ollus, M. (eds.) Methods and Tools for Collaborative Networked Organizations, pp. 119-154. Springer, Heidelberg (2008) 